\title{
Impact du changement climatique sur les vagues et la dérive littorale le long du littoral aquitain
}

\author{
Elodie CHARLES ${ }^{1}$, Déborah IDIER ${ }^{1}$
}

\author{
Pascale DELECLUSE ${ }^{(2)}$, Michel DEQUE(2), Gonéri LE COZANNET ${ }^{(1)}$ \\ 1.BRGM : elodie.charles@csiro.au ; d.idier@brgm.fr ; g.lecozannet@brgm.fr \\ 2.Météo-France / Centre National de Recherches Météorologiques : pascale.delecluse@meteo.fr ; michel.deque@meteo.fr
}

\begin{abstract}
RÉSUMÉ. - La dynamique sédimentaire littorale est contrôlée par un ensemble de facteurs hydrodynamiques qui sont susceptibles d'être modifiés par le changement climatique. Les vagues ont un rôle dominant dans la dynamique du littoral aquitain. Afin d'analyser l'impact du changement climatique, un modèle de vagues est mis en place et des simulations sont réalisées pour les climats actuel (1961-2000) et futurs (2061-2100). L'analyse des futures conditions de vague dans le Golfe de Gascogne met en évidence une diminution généralisée des hauteurs de vague et une rotation horaire de l'ensemble des vagues estivales et des houles hivernales par rapport au climat actuel. Le long de la côte aquitaine, la diminution des hauteurs de vague est toujours significative. Cependant, la réfraction bathymétrique induit une atténuation de la rotation des vagues, en particulier des houles hivernales. Le calcul de la dérive littorale (aussi appelée flux de sédiment longshore) au niveau de la plage de Biscarrosse à partir de formules empiriques indique que ces changements résulteraient en une diminution des flux longshore pouvant atteindre $10 \%$. Cette diminution est associée à la diminution des hauteurs de vague à proximité de la côte. Enfin, l'inventaire des incertitudes liées aux différentes étapes de cette étude suggère des pistes d'amélioration de la quantification des futurs flux de sédiment, par exemple en considérant davantage de modèles climatiques ou en modélisant les vagues dans la zone de déferlement.
\end{abstract}

Mots clefs : Vagues, Changement climatique, Transport sédimentaire, Côte aquitaine, Modélisation

\section{Climate change impact on waves and longshore sediment flux of the Aquitanian coast}

\begin{abstract}
Coastal sediment dynamics are controlled by a set of hydrodynamics factors likely to be modified by climate change. Waves are a dominant factor in the dynamics of the Aquitanian coast. In order to analyse the climate change impact, we set up a wave model and carried out simulations for present (1961-2000) and future (2061-2100) climates. The analysis of future wave conditions in the Bay of Biscay highlights a generalized decrease of wave heights and a clockwise rotation of summer waves and winter swell, relatively to present climate. Along the Aquitanian coast, the wave height decrease is also significant. However, bathymetric refraction induces a reduction of the clockwise rotation, particularly in winter swell. The calculation of longshore sediment fluxes at the Biscarrosse beach, using empirical formulae, shows that the projected wave changes would result in a decrease of longshore fluxes (up to $10 \%$ ). This decrease is associated with wave height decrease in the coastal area. Finally, the listing of the uncertainties related to the different steps of this study suggests potential tracks to improve the future sediment fluxes assessment, for instance by considering a larger number of climate models or by modelling waves in the breaking zone.
\end{abstract}

Key words : waves, climate change, sediment transport, Aquitanian coast, modelling

\section{INTRODUCTION}

Le changement climatique est susceptible de modifier les caractéristiques actuelles du littoral. Bordée par le Golfe de Gascogne, la côte aquitaine est principalement composée de plages sableuses. L'évolution de ces plages est liée aux variations spatiales des flux de sédiment perpendiculaires à la plage, mais aussi parallèles à la plage, appelés dérive littorale ou flux longshore. Ces flux longshore résultent de l'action des vagues. La figure $1 \mathrm{~b}, \mathrm{c}, \mathrm{d}$ illustre ce mécanisme ainsi que la dépendance de ces flux aux directions et hauteurs de vague. On peut noter que l'augmentation de l'angle d'incidence des vagues peut induire soit une augmentation des flux (si cet angle est inférieur à environ $45^{\circ}$ ), soit une diminution (si cet angle est supérieur à $45^{\circ}$ ). Pour le cas de la côte aquitaine, cet angle est généralement inférieur à $45^{\circ}$.
Les flux de sédiment sont par ailleurs soumis aux évolutions des sources de sédiment et des actions anthropiques pouvant modifier ces flux, comme par exemple les ouvrages perpendiculaires à la côte (épis). Des études antérieures (LCHF, 1979) ont permis de caractériser les flux longshore le long de la côte aquitaine : un flux vers le Nord au Sud de la Gironde, puis un flux orienté vers le Sud, jusqu'à l'Adour où ce flux peut ponctuellement s'inverser (figure 1a). Le changement climatique, en modifiant les champs de vent à l'échelle du bassin Atlantique nord, modifiera donc les champs de vague qui contribuent au flux sédimentaire longshore. Le présent article vise ainsi à apporter des éléments de réponse aux questions suivantes : (1) quels sont les effets du changement climatique sur les climats de vague futurs ? (2) en quoi ces modifications pourront-elles affecter les flux sédimentaires longshore? 


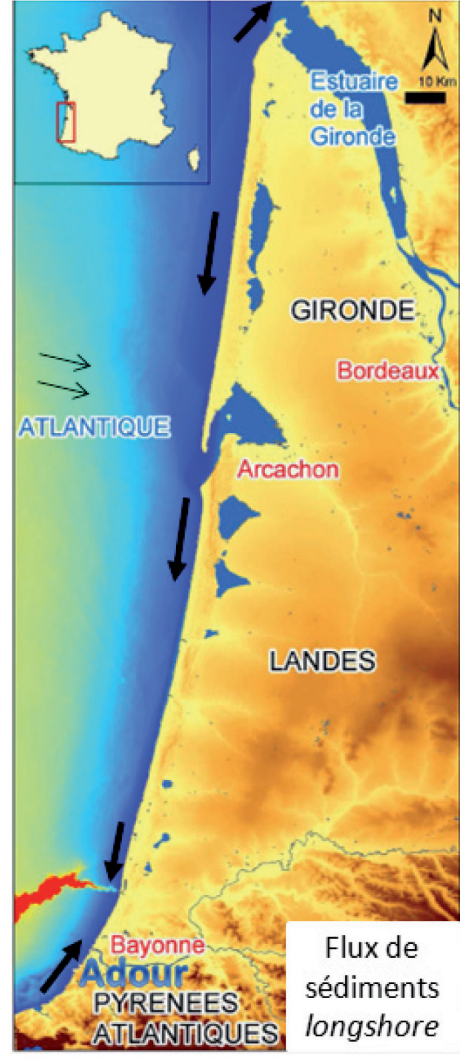

(a)

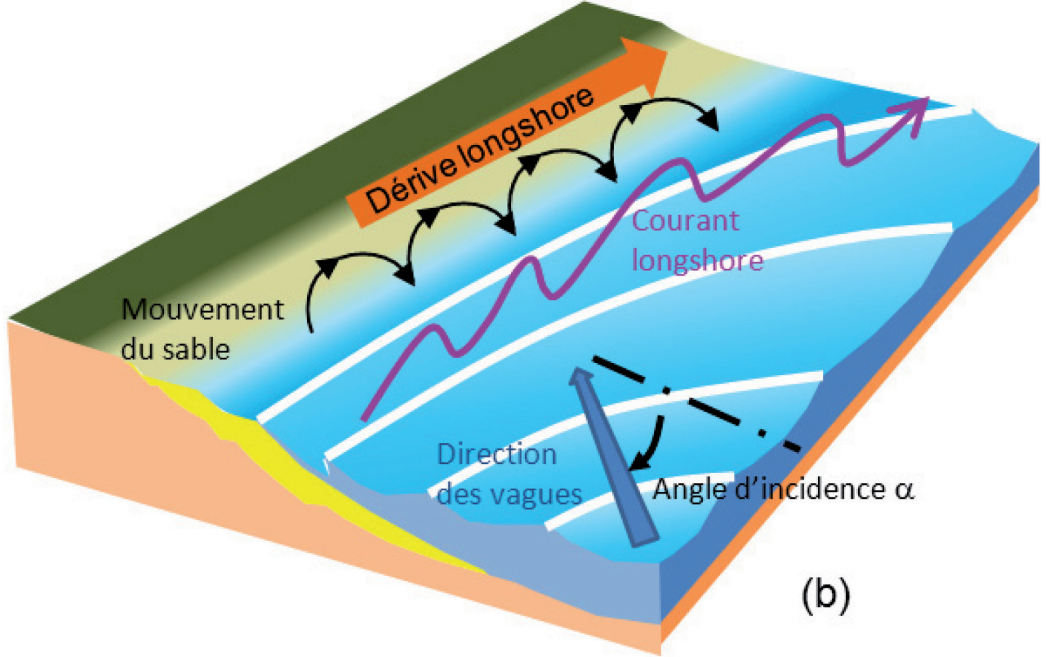

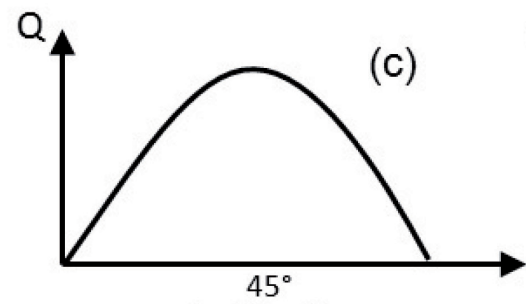

Angle d'incidence

au déferlement $\alpha_{b}$

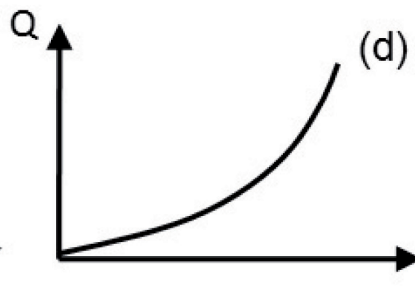

Hauteur de vagues au déferlement $\mathrm{Hb}$

Figure 1 : Côte Aquitaine et transport de sédiment littoral longshore. (a) : Schéma des flux sédimentaires littoraux longshore dominants (flèches épaisses) le long de la côte aquitaine, adapté de LCHF (1979). $\rightarrow:$ direction des vagues dominante. Fond de carte issu de Mugica et al. (2008), sources : BD-TOPO IGN, SHOM. (b) : Schéma des mécanismes induisant le transport longshore des sédiments, adapté des schémas du cours d'océanographie physique de The University of Western Ontario (http :// instruct.uwo.ca) (c,d) : schémas traduisant la dépendance des flux de sédiment longshore $Q$ en fonction de l'angle d'incidence (c) et de la hauteur (d) des vagues au déferlement, respectivement à hauteur et à angle constants.

Les analyses existantes des futures conditions de vague portent principalement sur les hauteurs de vague à l'échelle mondiale. Elles sont basées sur des projections de vagues présentant une résolution spatiale faible (supérieure à $1^{\circ}$ ) et indiquent des résultats très variables dans le Golfe de Gascogne. Quelques analyses à résolution spatiale plus fine (inférieure à $0.5^{\circ}$ ) prédisent soit une variation non significative, soit une diminution des hauteurs de vague dans le Golfe de Gascogne (Debernard \& Roed, 2008 ; Zacharioudaki et al., 2011). Cependant, d'autres paramètres de vague, à savoir leurs période et direction sont nécessaires pour étudier la dynamique littorale.

Une étude régionale des conditions de vague futures a été réalisée afin d'évaluer dans un premier temps les changements potentiels des conditions de vague dans le Golfe de Gascogne et d'analyser dans un second temps l'évolution des flux de sédiment longshore liés aux vagues le long de la côte aquitaine. Cette étude se base sur des projections de vagues réalisées pour le Golfe de Gascogne, présentant une résolution spatio-temporelle adaptée à l'analyse des conditions de vague du large à la côte (jusqu'à une profondeur d'environ $20 \mathrm{~m}$ ).

\section{MÉTHODE ET DONNÉES}

Les conditions de vague dans le Golfe de Gascogne et le long de la côte aquitaine sont issues de la base de données
BoBWA-10kF (Bay of Biscay Wave Atlas - Forecast, avec une résolution spatiale de $10 \mathrm{~km}$, Charles et al., 2012b). Les hauteurs, périodes et directions de vague sont disponibles pour un scénario de référence (REF) présentant les mêmes concentrations en gaz à effet de serre et aérosols que celles observées sur la période 1961-2000 et pour trois scénarios futurs (A2, A1B et B1) (Nakicenovic et al., 2000) sur la période 2061-2100. Les vagues issues de cette base de données ont été simulées avec le modèle spectral de vagues WAVEWATCH III (WW3, Tolman 2009), avec le paramétrage d'Ardhuin et al. (2010). Elles ont été modélisées sur deux domaines emboîtés (Figure 2), l'Atlantique Nord $\left(\mathrm{dx}=0.5^{\circ}\right)$ et le Golfe de Gascogne $\left(\mathrm{dx}=0.1^{\circ}\right)$, à partir de champs de vent projetés par le modèle ARPEGEClimat (Gibelin et Déqué, 2003). Une correction de type quantile-quantile (Déqué, 2007) a été réalisée sur les hauteurs, périodes et directions de vague, afin de corriger les biais systématiques du modèle climatique. Cette correction a été construite en comparant les distributions statistiques des vagues issues de BoBWA-10kF-REF à celles des vagues issues de BoBWA-10kH (Bay of Biscay Wave Atlas - Hindcast, avec une résolution spatiale de $10 \mathrm{~km}$, Charles et al., 2012a). Dans la suite de cette étude, nous utilisons les paramètres corrigés de hauteur significative (Hs), de période moyenne (Tm-10) et de direction moyenne (Dm) issues de BoBWA-10kF, sur le domaine couvrant le Golfe de Gascogne $\left(0.1^{\circ} \mathrm{x} 0.1^{\circ}\right.$, toutes les 6 heures $)$ et au niveau des bouées Gascogne $\left(5^{\circ} \mathrm{W}-45.201^{\circ} \mathrm{N}\right.$, profondeur : $4500 \mathrm{~m}$, 


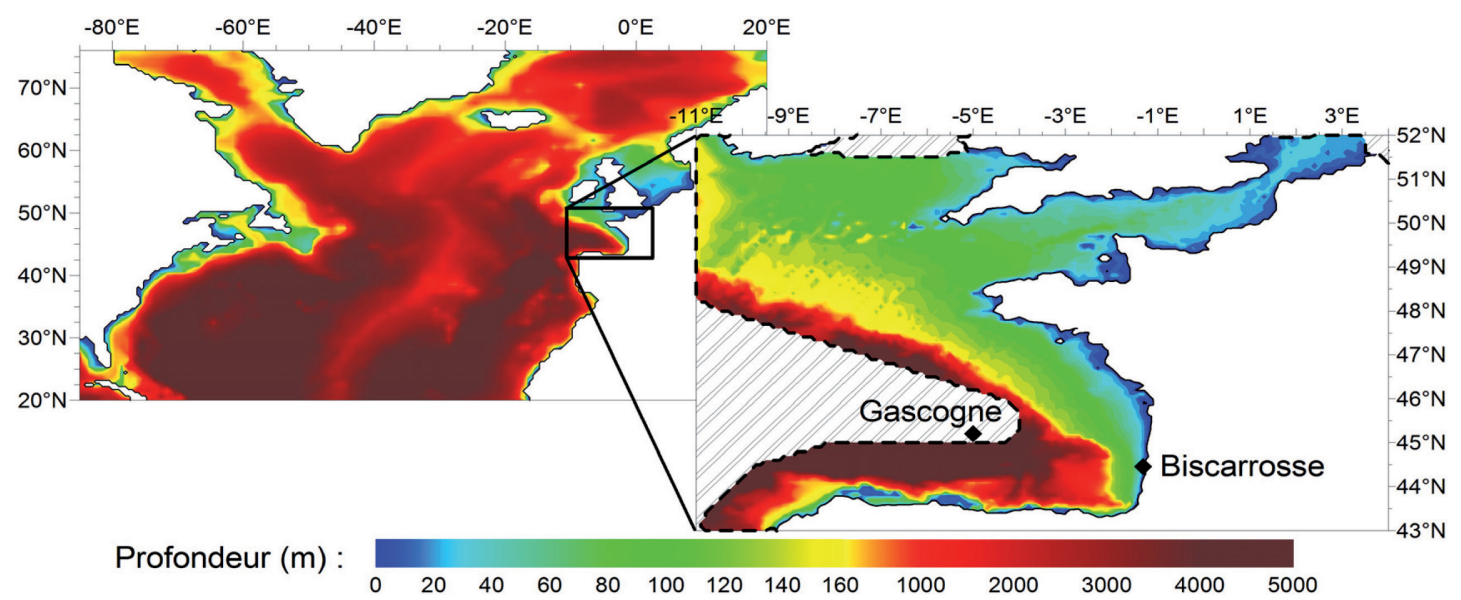

Figure 2 : Domaines utilisés pour modéliser les vagues avec WW3. Les zones hachurées (profondeurs supérieures à $4000 \mathrm{~m}$ ) sont exclues du second domaine. Les positions des bouées Gascogne et Biscarrosse sont indiquées par des diamants.

issue du rang 1) et Biscarrosse $\left(1.32^{\circ} \mathrm{W}-44.46^{\circ} \mathrm{N}\right.$, profondeur : $26 \mathrm{~m}$, issue du rang 2) (toutes les heures).

\section{ANALYSE DU CLIMAT DE VAGUE FUTUR}

Les changements des conditions de vague dans le Golfe de Gascogne et le long de la côte aquitaine sont analysés pour l'hiver (déc., jan., fév.) et l'été (juin, juil., août). On s'intéresse en particulier au scénario A2, qui présente un taux d'émissions de gaz à effet de serre plus élevé que les scénarios $\mathrm{A} 1 \mathrm{~B}$ et $\mathrm{B} 1$, et donc des changements plus marqués par rapport au climat actuel. Les conditions de vague du scénario REF sur la période 1961-2000 sont comparées à celles du scénario A2 sur la période 2061-2100. Dans un premier temps, les changements sont analysés à l'échelle régionale, sur le domaine du Golfe de Gascogne, puis dans un second temps à l'échelle locale, au niveau d'une bouée hauturière et d'une bouée côtière.

A l'échelle régionale (Figure 3), on peut noter les changements suivants dans le Golfe de Gascogne : une diminution généralisée des hauteurs de vague (jusqu'à $-20 \mathrm{~cm}$ en été), une rotation horaire des directions de vague en été $\left(\right.$ de $3^{\circ}$ à $\left.10^{\circ}\right)$ et une légère diminution des périodes en été (environ $-0.5 \mathrm{~s}$ ).

Ces changements moyens sont la somme des changements de plusieurs types de vague (houle, mer de vent, vagues intermédiaires). Afin de mieux caractériser leurs évolutions, une étude approfondie est réalisée en deux points : au niveau de la bouée Gascogne ( $\sim 500 \mathrm{~m}$ de hauteur d'eau), située au centre du Golfe de Gascogne, et au niveau de la bouée Biscarrosse ( $26 \mathrm{~m}$ de hauteur d'eau), située le long de la côte aquitaine. Les diagrammes bivariés (Figure 4) des conditions de vague pour le climat présent (scénario REF,
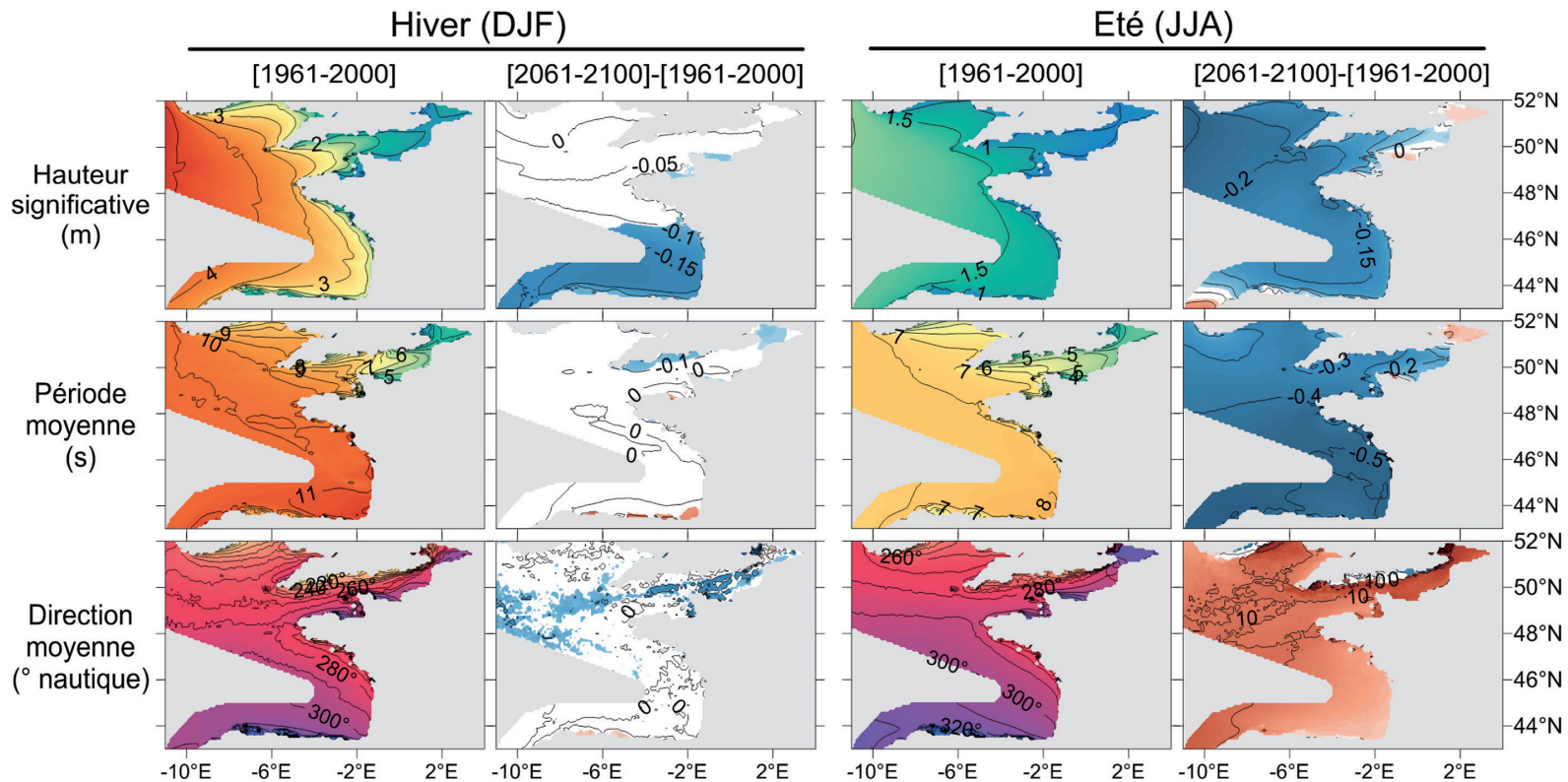

Figure 3 : Cartes des conditions de vague actuelles moyennes et des changements prédits pour le futur. Les hauteurs, périodes et directions moyennes pour le scénario présent REF (colonnes 1 et 3), et les différences entre le scénario futur A2 (2061-2100) et le scénario présent REF (1961-2000) (colonnes 2 et 4) sont données pour l'hiver et l'été (adapté de Charles et al., 2012b). Dans les colonnes 2 et 4 , seules les zones pour lesquelles les changements sont significatifs à plus de $95 \%$ (T-Test de Student) sont colorées. 

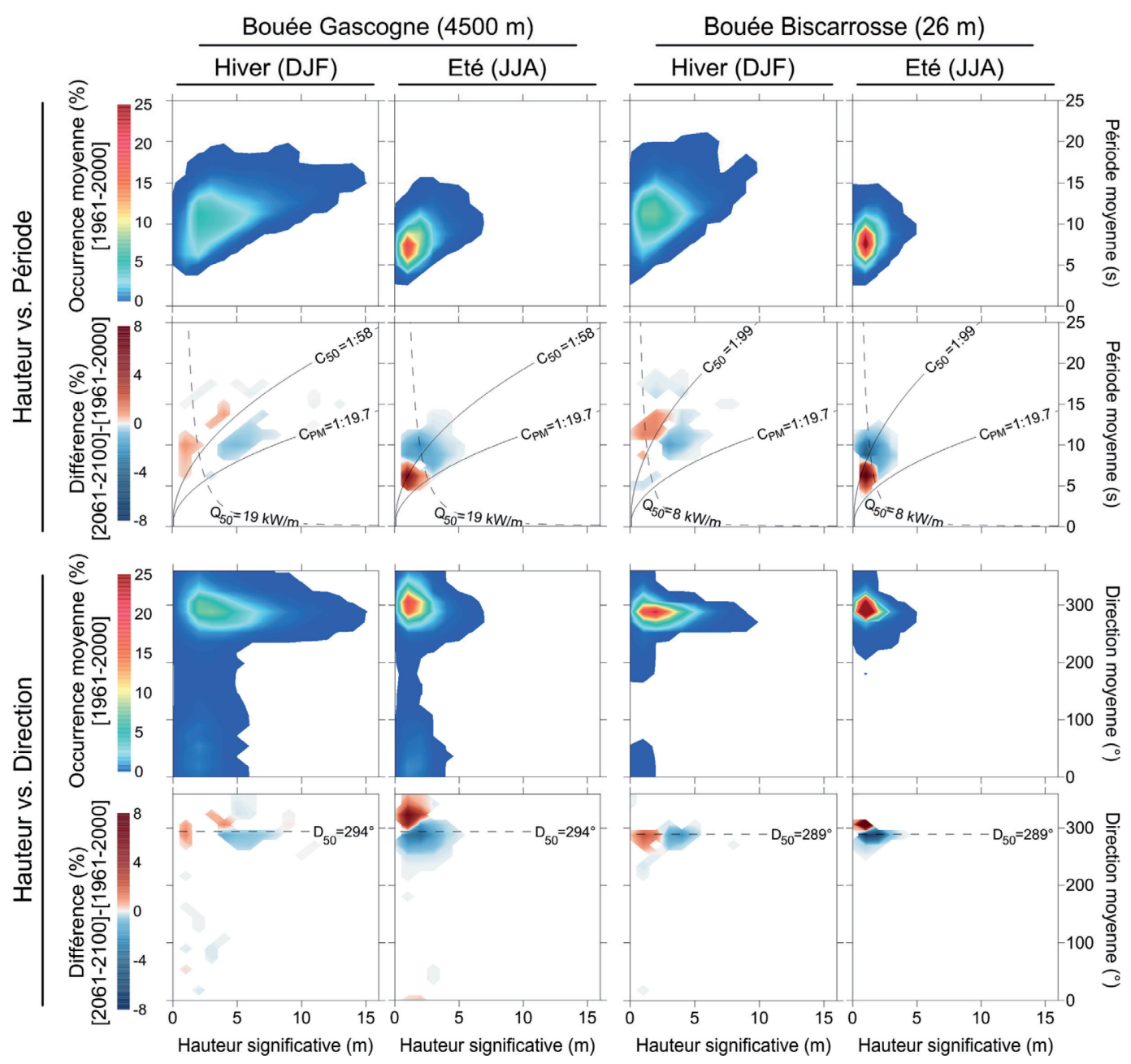

Figure 4 : Diagrammes bivariés des conditions de vague au large et à la côte et changements prédits pour le futur. Les diagrammes bivariés (adaptés de Charles et al., 2012b) au niveau des bouées Gascogne (gauche) et Biscarrosse (droite) représentent les occurrences de conditions de vague pour le scénario présent REF et les changements entre le scénario futur A2 (2061-2100) et le scénario présent REF (1961-2000) pour l'hiver et l'été. Seuls les changements significatifs à plus de $95 \%$ (T-Test de Student) sont tracés. Dans la $2^{e}$ rangée, les lignes continues correspondent à la cambrure médiane annuelle $c_{50}$ et à la cambrure de Pierson-Moskovitz $c_{P M}$, la ligne pointillée correspond à la valeur médiane annuelle du flux d'énergie $Q_{50}$. Dans la $4^{e}$ rangée, la ligne pointillée correspond à la direction médiane annuelle $D_{50}$.

1961-2000) permettent de visualiser les variations du climat de vague suivant la saison et suivant la distance à la côte. On peut ainsi noter qu'au niveau de la bouée côtière Biscarrosse, les effets bathymétriques induisent une réduction des hauteurs de vague et un resserrement des directions autour de la normale à la côte.

Concernant les changements entre les conditions de vague du climat futur (scénario A2, 2061-2100) et du climat présent (scénario REF, 1961-2000), on note une évolution différente en été et en hiver (Figure 4). Les valeurs médianes de la cambrure c, du flux d'énergie Q et de la direction Dm sont calculées à partir des données hivernales et estivales de REF au niveau de chaque bouée et sont reportées sur les diagrammes bivariés correspondants afin d'en faciliter l'analyse. Le tableau 1 résume la méthode et la terminologie choisies pour désigner les vagues en fonction de leur cambrure ou de leur flux d'énergie. D'une manière générale, en été, les vagues dont la provenance est plus méridionale que la moyenne deviennent moins fréquentes. En hiver, ce phénomène est moins marqué au niveau de la bouée Gascogne, et il n'est pas visible au niveau de la bouée Biscarosse (Figure 4). En été, au niveau des deux bouées, l'occurrence des vagues énergétiques diminue, et l'occurrence des vagues peu énergétiques augmente. En hiver, au niveau des deux bouées, l'occurrence de la houle augmente, tandis que l'occurrence des vagues intermédiaires diminue.

Ces changements de conditions de vague locales sont produits par des changements de conditions de vent sur différentes zones (Atlantique Nord, Golfe de Gascogne). L'analyse détaillée des relations entre changements des conditions de vent et de vague a été réalisée par ailleurs (Charles et al., 2012b). Considérons ici par exemple la rotation horaire globale des directions de vague observée en été, via l'analyse de la différence d'occurrence des vagues (visible sur la Figure 4, ligne 4). Une rotation horaire des directions de vent à l'échelle du golfe de Gascogne engendre un changement de direction de la mer de vent à l'échelle locale (Biscarosse). A l'échelle régionale, il existe aussi une rotation horaire des directions de vent au Nord et à l'Ouest du Golfe de Gascogne (les vents étant davantage orientés vers le Sud pour le climat futur), impliquant davantage de vagues se propageant du Nord vers le Sud, et donc entrant dans le Golfe de Gascogne par le Nord et moins par l'Ouest. Enfin, à l'échelle océanique, on note une diminution des 
Tableau 1 : Méthode choisie de répartition des vagues. La classification est basée sur la cambrure des vagues ( $c=2 \pi H_{s} / g T_{m}^{2}$ avec $T_{m}$ la période moyenne, $g$ l'accélération de pesanteur et $H_{s}$ la hauteur de vague significative) ou le flux d'énergie ( $Q=\rho T_{m} g^{2} H_{s}^{2} / 64 \pi$ avec $\rho$ la densité de l'eau). Pour plus de détails, voir (Charles et al., 2012a).

$L^{\prime}$ indice 50 indique la valeur médiane annuelle et $c_{P M}$ correspond à la cambrure de Pierson-Moskovitz $\left(c_{P M}=1: 19.7\right)$.

\begin{tabular}{|l|l|}
\hline \multicolumn{1}{|c|}{ Cambrure } & \multicolumn{1}{c|}{ Type de vague } \\
\hline $\mathrm{c}<\mathrm{c}_{50}$ & Houle \\
\hline $\mathrm{c}_{50}<\mathrm{c}<\mathrm{c}_{\mathrm{PM}}$ & Vagues intermédiaires \\
\hline $\mathrm{c} \approx \mathrm{c}_{\mathrm{PM}}$ & Mer de vent \\
\hline
\end{tabular}

\begin{tabular}{|l|l|}
\hline \multicolumn{1}{|c|}{ Flux d'énergie } & \multicolumn{1}{c|}{ Type de vague } \\
\hline $\mathrm{Q}<\mathrm{Q}_{50}$ & Vagues peu énergétiques \\
\hline $\mathrm{Q}>\mathrm{Q}_{50}$ & Vagues énergétiques \\
\hline
\end{tabular}

vitesses de vent dans certaines zones de l'Atlantique Nord, engendrant une réduction de l'occurrence des houles d'Ouest et d'Ouest-Nord-Ouest.

\section{EFFETS DES CHANGEMENTS DE CONDITIONS DE VAGUE SUR LA DÉRIVE LITTORALE}

Il est possible d'estimer les ordres de grandeur des flux longshore de sédiment à partir des conditions de vague. En particulier, plus les hauteurs de vague sont fortes et/ou plus l'angle d'incidence des vagues est fort (en restant inférieur à $45^{\circ}$, CERC, 1984), plus les flux sont forts (Figure $1 \mathrm{c}, \mathrm{d}$ ). Or, l'analyse précédente montre que les hauteurs et directions des vagues au large devraient être affectées par le changement climatique. En outre, les résultats des simulations montrent que la diminution des hauteurs de vague identifiée au large entre climat présent et climat futur se propage jusqu'à la côte, tout comme les changements de direction affectant les petites vagues, bien qu'ils soient atténués par la réfraction bathymétrique. Ainsi, dans cette partie, nous analysons dans quelle mesure ces changements pourraient affecter de manière significative les flux longshore à l'horizon 2100. En particulier, plus que de quantifier finement les flux et leur évolution future, il s'agit ici d'identifier si les changements climats de vagues présentés ci-dessus contribueraient à une augmentation ou une diminution des flux de sédiments longshore.

Une analyse de l'évolution temporelle des flux de sédiment longshore a été réalisée pour la plage de Biscarrosse, en utilisant les conditions de vague simulées au niveau de la bouée Biscarrosse. Plusieurs formulations permettent d'estimer les ordres de grandeur de ces flux à partir des conditions de vague. Globalement, elles dépendent toutes explicitement de la hauteur des vagues et de leur incidence. Certaines, comme celle de Kamphuis (1991) intègrent aussi explicitement la pente de la plage, la taille de grain, ou la période des vagues. Cependant, comme rappelé par Pilkey et Cooper (2002), il existe une forte incertitude dans les résultats obtenus à partir de formules de transport longshore et, au mieux, seuls les ordres de grandeur sont à considérer.

Dans un premier temps, une estimation préliminaire de l'ordre de grandeur des flux absolus est effectuée en utilisant les formules du LCHF (1979) et de Kamphuis (1991). En effet, ce sont des formules qui apparaissent adaptées, entre autres, aux côtes Atlantique aquitaine et portugaises (Cayocca, 1996 ; Abadie et al., 2006 ; Balouin et al., 2005). Ces formules utilisent les conditions de vague au point de déferlement. Celles-ci sont obtenues en utilisant le jeu de données de vagues modélisé au large de Biscarrosse (1961-2000) et en y appliquant les lois de Snell et de conservation d'énergie, et ce, en faisant donc l'hypothèse que pour le cas étudié les contours bathymétriques sont rectilignes et parallèles ainsi qu'en négligeant la dissipation de l'énergie avant le déferlement. Ainsi, les formules du LCHF (1979) et de Kamphuis (1991) appliquées à ces données de vagues au déferlement donnent des flux de sédiment de l'ordre respectivement de 160000 et $200000 \mathrm{~m}^{3} / \mathrm{an}$. On peut noter que ces estimations sont du même ordre de grandeur que celles d'Abadie et al. (2006) - $100000 \mathrm{~m}^{3} / \mathrm{an}$, valeur estimée à environ $40 \mathrm{~km}$ au sud de Biscarrosse - et de LCHF (1987) - $520000 \mathrm{~m}^{3} / \mathrm{an}$, valeur estimée à Mimizan (situé à environ $25 \mathrm{~km}$ au sud de Biscarrosse). Parmi les pistes à explorer pouvant expliquer les différences obtenues, outre la localisation spatiale exacte, on peut noter que les données de vagues en entrée diffèrent en nature et période couverte, avec un jeu de 40 ans de données modélisées pour la présente étude, 8 ans (1997-2005) de données modélisées (NOAA-WW3) corrigées pour Abadie et al. (2006) et des données observées sur une période antérieure à 1985 pour les estimations du LCHF (1987). Toutefois, les flux estimés restent du même ordre de grandeur $\left(\mathrm{O}\left(100000 \mathrm{~m}^{3} / \mathrm{an}\right)\right)$.

Afin d'étudier la différence entre les flux actuels et les flux futurs, nous analysons les flux principalement en termes de valeur relative, soit par rapport au flux annuel (Figure 5.a), soit par rapport au flux du climat actuel correspondant (Figure 5.b). Les flux relatifs donnés sont la moyenne des valeurs obtenues avec les deux formules. La barre d'erreur indique les valeurs minimale et maximale obtenues en appliquant chacune de ces formules.

La Figure 5.a donne un aperçu de la variabilité saisonnière des flux de sédiment longshore. La quantité de sédiment déplacée est trois fois plus importante en hiver qu'en été. Les écarts entre les formules sont importants pendant ces deux saisons. Au printemps et en automne, les flux ont une amplitude similaire à la moyenne annuelle.

Pour le climat futur (Figure 5.b), nous nous intéressons en particulier à l'évolution des flux sur l'année, en hiver et en été. Quelle que soit la période de l'année, on constate que les changements sont d'autant plus importants que le scénario est émissif en gaz à effet de serre. Ainsi, les changements de vague projetés pour le scénario A2 donnent une réduction du flux de l'ordre de $10 \%$ par rapport au climat actuel. Seuls les résultats pour le scénario B1 montrent une légère augmentation des flux de sédiment longshore. Ces résultats indiquent que, à Biscarrosse, la baisse projetée des hauteurs de vague pour le climat futur serait le facteur prédominant dans l'évolution des flux de sédiment. La rotation horaire des vagues projetée, ayant pour effet d'intensifier les flux sédimentaires vers le Sud dans cette région, serait donc largement compensée par la diminution des hauteurs de vague. 
a)

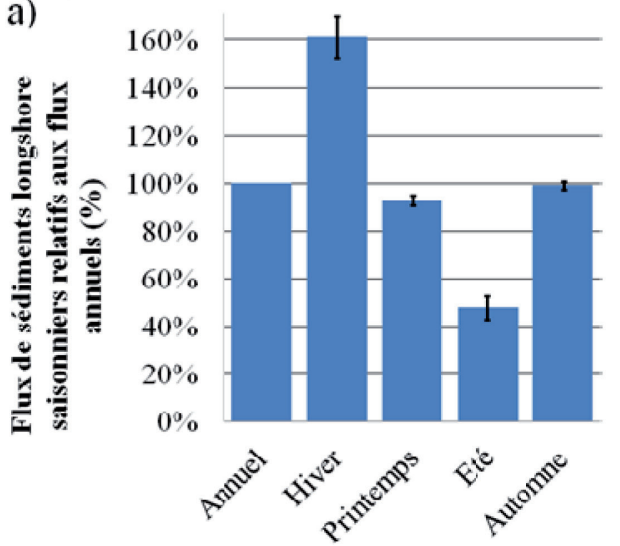

b)

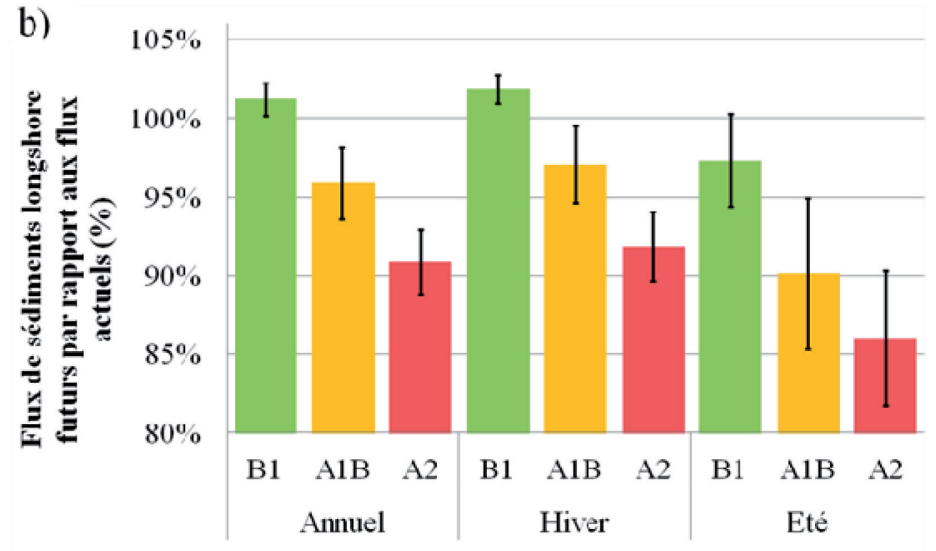

Figure 5 : Variations des flux de sédiment longshore pour les climats présent et futurs à Biscarrosse. a) Variations saisonnières des flux de sédiment longshore actuels (scénario REF), relativement au flux annuel, b) Flux de sédiment longshore pour trois scénarios futurs $(B 1, A 1 B, A 2)$, relativement au flux actuel pour la même saison.

\section{INCERTITUDES}

Les résultats présentés sont issus de différentes étapes (Figure 6), chacune de ces étapes comportant des incertitudes.

En effet, la première étape est le choix des scénarios. Ici, nous avons choisi trois scénarios $(\mathrm{B} 1, \mathrm{~A} 1 \mathrm{~B}, \mathrm{~A} 2)$ issus du GIEC (IPCC, 2007). Ces scénarios s'appuient sur la définition de conditions socio-économiques qui contraignent des niveaux d'émissions qui, transformés en concentrations, permettent ensuite d'établir les projections climatiques. Le choix des conditions socio-économiques, et donc des niveaux d'émission d'ici 2100, est un premier niveau d'incertitude. On peut noter que, dans le prochain exercice du GIEC, les scénarios seront construits à partir de profils représentatifs d'évolution de concentration, et non plus sur des conditions socio-économiques.

Une fois le scénario choisi, un modèle climatique est utilisé pour le traduire en champ de vent, indispensable pour calculer le champ de vague. Dans la présente étude, un seul modèle a été utilisé : ARPEGE-Climat. Afin d'estimer les incertitudes associées au choix du modèle climatique,

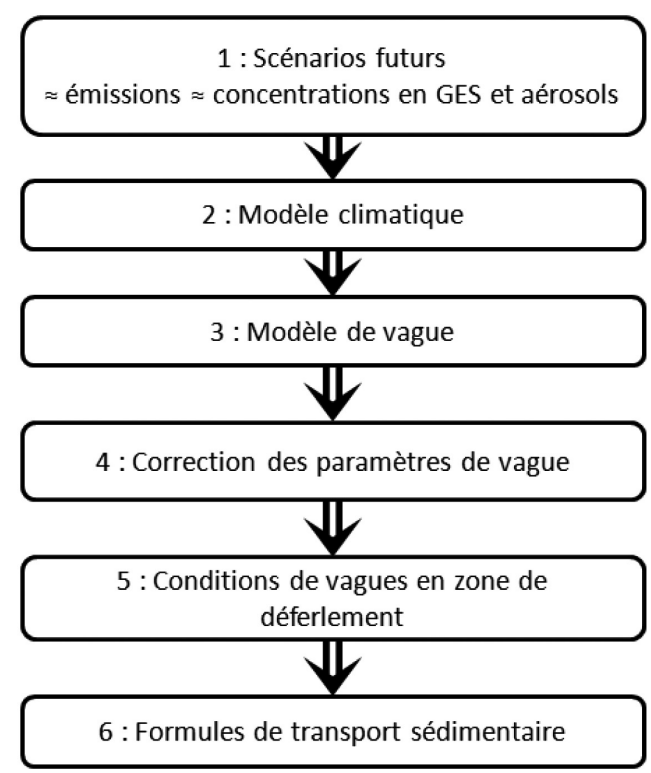

Figure 6 : Etapes (et incertitudes) principales aboutissant au calcul de flux de sédiment longshore. il serait pertinent d'utiliser plusieurs modèles climatiques (Grabemann et Weisse, 2008).

L'étape suivante est de modéliser les vagues à partir du champ de vent obtenu. Dans cette étude, nous avons utilisé le modèle WW3. De la même manière que pour les modèles climatiques, il peut être intéressant d'utiliser plusieurs modèles de vagues pour mieux estimer les incertitudes. Toutefois, dans la présente étude, le modèle utilisé a été validé de manière satisfaisante par rapport aux données observées (Charles et al, 2012a). On peut donc penser que les incertitudes associées à cette étape sont faibles au regard de celles des étapes précédentes.

Les modèles climatiques comportant des biais, les vagues modélisées ont été corrigées par une méthode quantilequantile, par comparaison aux vagues modélisées et validées sur la période 1958-2001 (Charles et al., 2012a, 2012b). Cependant, ce type de correction suppose une hypothèse de stationnarité dans les distributions. Aussi, pour compléter l'estimation des incertitudes, utiliser d'autres méthodes de correction pourrait être intéressant.

Une fois les conditions de vague obtenues, pour pouvoir calculer les flux de sédiment longshore à partir de formules empiriques, il est nécessaire d'estimer les conditions de vague au déferlement. Dans la présente étude, le calcul est basé sur une hypothèse de contours bathymétriques rectilignes et parallèles et sur la conservation de l'énergie avant le déferlement. Or, la côte Aquitaine est caractérisée par des barres sous-marines bidimensionnelles ou tridimensionnelles, qui évoluent temporellement et spatialement. Ces barres ne sont pas visibles dans la bathymétrie utilisée. Un modèle numérique de vagues de résolution d'une dizaine de mètres pourrait permettre de déterminer plus finement les caractéristiques de vague au déferlement. Toutefois, il faut noter que la position des barres et leur forme sont variables dans le temps. Ainsi, même en utilisant un modèle de vagues plus fin, cette étape soulèverait d'autres incertitudes.

Enfin, la dernière étape consiste à calculer les flux de sédiment longshore à partir des conditions de vague au déferlement, entre autres. Les barres d'erreurs de la figure 5 correspondent aux incertitudes liées au choix d'un modèle de transport longshore. Les modèles choisis ont été considérés comme valables sur la côte aquitaine. Les barres d'erreurs représentent donc une partie des incertitudes épistémiques, mais pas la totalité, en raison du fait que ces formules restent des représentations imparfaites des processus 
(Pilkey et Cooper, 2002). Dans le cas où l'on voudrait estimer de manière plus quantitative les flux de sédiments longshore, une modélisation plus fine des vagues, en décrivant précisément la bathymétrie jusqu'à la côte, est nécessaire. Toutefois, le site de Biscarosse est complexe avec la présence de barres sous-marines dont la forme et la position évoluent dans le temps : les barres externes migrent vers le large et deviennent linéaires suite aux épisodes de fortes houles, tandis qu'elles se rapprochent de la côte et prennent des formes tridimensionnelles pendant les périodes de temps calme. L'utilisation d'un modèle morphodynamique de plages à barres pourrait être envisagée, mais aujourd'hui aucun modèle numérique n'est capable de reproduire l'évolution réelle de la bathymétrie aux échelles pluri-décennales. En outre, dans cette étude, on s'intéresse à la contribution des vagues dans les évolutions futures de flux longshore. Une modélisation fine des vagues nécessiterait d'estimer ce que pourrait être la bathymétrie future. Or, comme l'ont montré Thiébot et al. (2012) et Brivois et al. (2012), le type de morphologie présente sur ce type de plage à barre est très sensible aux conditions de vagues. En particulier, pour des vagues modérés $(\mathrm{Hs}=1 \mathrm{~m})$, les modifications d'angle d'incidence des vagues peuvent engendrer des changements significatifs sur le type de morphologie présente en zone littorale (plage à barre en croissant, sans barre, à barre oblique, ...). Il est donc possible que la morphologie future diffère de la morphologie actuelle. Ainsi, l'estimation quantitative des flux longshore à l'échelle pluri-décennale à partir de modélisation fine des vagues sur un site à barres, et pour une estimation de flux longshore futurs, nécessite la mise en place d'une méthodologie spécifique.

La question des flux de sédiment cross-shore (orientés perpendiculairement à la plage) n'est pas traitée dans la présente étude. Cependant, dans la caractérisation de l'évolution morphologique long-terme de la côte, ce type de flux peut avoir un impact significatif. Les flux cross-shore orientés vers le large sont principalement activés sous l'action d'évènements extrêmes d'une durée de quelques heures à quelques jours. Pendant les périodes de temps calme, les flux cross-shore sont généralement orientés vers la plage et permettent de reconstituer les réserves de sédiment. Ces flux sont fortement liés à la bathymétrie complexe de la zone de déferlement (Castelle et al. 2007) et à des épisodes climatiques extrêmes qui ne sont pas toujours bien reproduits par les modèles. Ces processus sont donc délicats à quantifier et demeurent mal connus.

\section{CONCLUSIONS}

Au large, les conditions de vague projetées pour le scénario A2 indiquent une diminution généralisée des hauteurs de vague (de 10 à $20 \mathrm{~cm}$ ) et une rotation horaire de l'ensemble des vagues en été (de 3 à $10^{\circ}$ ) ainsi que des houles en hiver. A proximité de la côte, la diminution des hauteurs de vague est toujours significative. Cependant, la réfraction bathymétrique induit une atténuation de la rotation horaire des directions des vagues, en particulier pour les vagues les plus hautes, telles que les houles hivernales.

L'analyse de la dérive littorale au niveau de la plage de Biscarrosse suggère une diminution de l'amplitude des flux de sédiment le long de ce segment de la côte aquitaine. Ce résultat, obtenu en un point, ne permet pas de conclure à une tendance à l'érosion ou à l'accrétion sur ce segment, l'érosion ou l'accrétion étant liée aux variations spatiales de flux de sédiment. Cependant, ces résultats permettent de conclure que les changements de conditions de vague projetés sont suffisamment significatifs pour modifier les flux longshore du littoral aquitain. Une perspective à ce travail serait d'analyser l'évolution des flux de sédiment longshore sur la côte aquitaine dans son ensemble afin d'analyser ses effets potentiels sur l'évolution du trait de côte à la fin du $21^{\mathrm{e}}$ siècle.

\section{REMERCIEMENTS}

Ce travail a été réalisé dans le cadre d'une thèse co-encadrée par le BRGM et le CNRM/Météo-France, bénéficiant d'une bourse de thèse du Fonds AXA pour la Recherche. Les auteurs remercient F. Ardhuin, B. Castelle, R. Pedreros et C. Mallet pour leurs contributions.

\section{REFERENCES}

Abadie S., Butel R., Mauriet S., Morichond D., Dupuis H. (2006) - Wave climate and longshore drift on the South Aquitaine coast. Continental Shelf Research. 26 : 1924-1939

Ardhuin F., Rogers E., Babanin A., Filipot J. F., Magne R., Roland A., Westhuysen A. V. D., QuefFeulou P., LefeVre J. M., Aouf L., Collard F. (2010) - Semi-empirical dissipation source functions for ocean waves : Part I, definition, calibration and validation. doi :10.1175/2010JPO4324.1. J. Phys. Oceanogr. 40 : 1917-1941

Balouin Y., Howa H., Pedreros R., Michel D. (2005) Longshore Sediment Movements from Tracers and Models, Praia de Faro, South Portugal. Journal of Coastal Research. 21(1) : 146-156

Brivois O., Idier D., Thiébot J., Castelle B., Le Cozannet G., Calvete D. (2012) - On the use of linear stability model to characterize the morphological behaviour of a double bar system. Application to Truc Vert Beach (France). C.R. Geoscience. 344 : 277-287

Castelle B., Bonneton P, Dupuis H., Sénéchal N. (2007) Double bar beach dynamics on the high-energy mesomacrotidal French Aquitanian coast : A review. doi :10.1016/j. margeo.2007.06.001. Mar. Geol. 245 : 141-159

CAYOCCA F. (1996) - Modélisation morphodynamique d'une embouchure tidale : application aux passes d'entrée du bassin d'Arcachon. Thèse de doctorat, Univ. Bdx 1.

Charles E., Idier D., Thiébot J., Le Cozannet G., Pedreros R., Ardhuin F., Planton S. (2012a) - Present wave climate in the Bay of Biscay : spatiotemporal variability and trends from 1958 to 2001. doi :10.1175/JCLI-D-11-00086.1. J. Climate. 25 : 2020-2035

Charles E., Idier D., Delecluse P., Déqué M., Le Cozannet G. (2012b) - Climate change impact on waves in the Bay of Biscay, France. doi :10.1007/s10236-012-0534-8. Ocean Dynamics. 62(6) : 831-848

CERC (1984) - Shore Protection Manual. 4th ed., 2 vols. Technical report, U.S. Army Engineer Waterways Experiment Station. Washington D.C. :U.S. Gov. Printing Office.

Debernard J. B., Roed L.P. (2008) - Future wind, wave and storm surge climate in the Northern Seas : a revisit. doi :10.1111/j.16000870.2008.00312.x. Tellus A. 60(3) : 427-438

DÉQUÉ M. (2007) - Frequency of precipitation and temperature extremes over France in an anthropogenic scenario : model results and statistical correction according to observed values. doi :10.1016/j.gloplacha.2006.11.030. Glob Planet Change. 57(1-2) : 16-26

GiBelin A.-L., DÉQuÉ M. (2003) — Anthropogenic climate change over the Mediterranean region simulated by a global variable resolution model. Clim. Dynam. 20 : 327-339 
Grabemann I., Weisse R. (2008) - Climate change impact on extreme wave conditions in the North Sea : an ensemble study. doi :10.1007/s10236-008-0141-x. Ocean Dynamics. 58 : 199-212

IPCC AR4 SYR (2007) — Climate Change 2007 : Synthesis Report Contribution of Working Groups I, II and III to the Fourth Assessment Report of the Intergovernmental Panel on Climate Change, Core Writing Team ; Pachauri, R.K ; and Reisinger, A., ed., IPCC, ISBN 92-9169-122-4.

KAmphuis J. W. (1991) - Alongshore sediment transport rate doi :10.1061/(ASCE)0733-950X(1991)117 :6(624). Journal of Waterway, Port, Coastal, and Ocean Engineering. 117(6) : 624-640

LCHF (1979) - Etude en nature de la côte aquitaine (entre la Pointe de la Grave et l'embouchure de l'Adour). Rapport établi pour la mission interministérielle pour l'aménagement de la côte aquitaine, Laboratoire Central Hydraulique de France.

LCHF (1987) - Catalogue sédimentologique des côtes françaises De la Baie du Mont-Saint-Michel à la frontière espagnole. partie $B$. Collection de la DER d'EdF $n^{\circ} 65$, Paris : Eyrolles.
Mugica J., Mallet C., Hennequin V. (2008) — Etude de l'évolution géomorphologique récente de la côte sableuse aquitaine, Rapport nBRGM/RP-56874-FR, 29 ill., 9 ann., 165

NAKicenovic N. ET AL. (2000) — Special Report on Emissions Scenarios : A Special Report of Working Group III of the Intergovernmental Panel on Climate Change, Cambridge University Press, Cambridge, U.K. 599

Pilkey O.H., Cooper J.A.G. (2002) - Longshore Transport Volumes : A Critical View. J. of Coast. Res. SI 36 : 572-580

Thiébot J., Idier D., Garnier R., Falqués A., Ruessink G. (2012) - The influence of wave direction on the morphological response of a double sandbar system. Continental Shelf Research. $32: 71-85$

Tolman H.L. (2009) - User manual and system documentation of WAVEWATCH III version 3.14. - Technical Note 276, NOAA / NWS / NCEP / MMAB.

Zacharioudaki A., Pan S., Simmonds D., Magar V., Reeve D. E. (2011) - Future wave climate over the west-European shelf seas. doi :10.1007/s10236-011-0395-6. Ocean Dynamics. 6 : $807-827$ 